\title{
INCLUSÃO DE RESTRIÇÕES DINÂMICAS NO PROBLEMA DE PLANEJAMENTO DE POTÊNCIA REATIVA
}

\author{
Edimar J. de Oliveira* \\ edimardacee.ufjf.br \\ Nelson Martins ${ }^{\dagger}$ \\ nelson@cepel.org
}

\author{
Rafael M. Fontoura* \\ rm_fontoura@yahoo.com
}

Leonardo Willer de Oliveira*

leonardowillerdyahoo.com

\author{
José L. R. Pereira* \\ jluiz@ieee.org \\ *Universidade Federal de Juiz de Fora-MG \\ ${ }^{\dagger}$ Centro de Pesquisas de Energia Elétrica - CEPEL-RJ
}

\section{RESUMO}

Este artigo apresenta uma nova metodologia para o planejamento da operação dos Sistemas Elétricos de Potência (SEP), na qual restrições dinâmicas de impactos em geradores são incorporadas ao problema de Fluxo de Potência Ótimo (FPO). Neste novo modelo, o transitório no instante da contingência é incorporado ao FPO como uma restrição operacional a fim de manter os impactos nos eixos dos geradores nos níveis recomendados pelo Operador Nacional do Sistema (ONS). Desta forma o tradicional problema de planejamento de potencia reativa incorpora os impactos nos eixos dos geradores do SEP quando ocorre uma contingência. O problema é formulado usando a metodologia primal-dual de pontos interiores associada à técnica de decomposição matemática de Benders. Estudos de casos com os sistemas IEEE-14 e IEEE-118 são realizados para mostrar o desempenho da metodologia proposta.

PALAVRAS-CHAVE: Planejamento da Operação, Restrições Dinâmicas, Análise de Contingências, Fluxo de Potencia Ótimo, Pontos Interiores, Decomposição de Benders.

\footnotetext{
Artigo submetido em 19/08/2006

1a. Revisão em 25/01/07

2a. Revisão em 14/04/07

3a. Revisão em 01/06/07

Aceito sob recomendação do Editor Associado Prof. Carlos A. Castro
}

\begin{abstract}
This paper presents a new methodology for power systems operation planning in which dynamic constraints related to the rotor shaft impacts are incorporated into the optimal power flow problem. In this new model the transients at the instant of the contingency is incorporated to the OPF problem, as an additional constraint, in order to keep the rotor shaft torques within limits, which are recommended by the National System Operator (ONS). Then the traditional problem of reactive power planning in electrical power systems includes the generator rotor shaft impact effects when a contingency is present. The problem is formulated using the primal-dual interior point technique associated to the mathematical Benders decomposition. Case studies using the IEEE-14 and IEEE-118 bus test systems are presented to show the effectiveness of the proposed methodology.
\end{abstract}

KEYWORDS: Operation Planning, Dynamics Constratints, Contingency Analysis, Optimal Power Flow, Interior Points, Benders Decomposition.

\section{INTRODUÇÃO}

O objetivo do planejamento da operação dos Sistemas Elétricos de Potência (SEP) é garantir uma operação ótima sob condições normais e operação viável sob regime de contin- 
gência (Monticelli et. al., 1987; Granville et. al., 1988). Para tanto, este planejamento consiste em determinar a localização e o valor do suporte de potência reativa necessários para a operação viável do sistema. O problema é colocado como um problema de FPO associado à minimização de suporte de potência reativa, considerando-se o sistema em contingência (Vaahedi et. al., 1999).

Tradicionalmente, a técnica de decomposição matemática de Benders (Benders, 1962) tem sido aplicada a este tipo de problema (Granville and Abib Lima, 1994). Para tanto, o problema é dividido em dois subproblemas hierárquicos: (i) subproblema de investimento, considerando-se operação do sistema em condições normais. Neste subproblema, os controles disponíveis são ajustados e os investimentos em fontes de potência reativa são realizados a fim de assegurarem uma operação viável do sistema em regime de contingência (Vaahedi et. al., 2001; Oliveira et. al., 2005); (ii) subproblema de operação, considerando-se o sistema em contingência. Neste subproblema é verificado se os ajustes e os investimentos realizados em (i) são suficientes para a operação viável. Caso não sejam suficientes, o sistema é inviável para operar com esta contingência. Neste caso, um corte de Benders é gerado para o subproblema de investimento. O processo continua até que a operação do sistema seja viável para toda a lista de contingências. A Figura 1 mostra esta decomposição em dois subproblemas.

Nesta figura, tem-se que:

$x_{1}$ São as variáveis de decisão dos ajustes e investimentos;

$x_{1}^{*}$ Representa o valor ótimo de $x_{1}$ calculado no subproblema de investimento;

$u\left(x_{1}\right)$ Representa a restrição de corte de Benders (Geoffrion, 1972) gerada no subproblema de operação.

Este tradicional problema de planejamento da operação ainda não incorpora na análise os impactos nos eixos dos geradores causados no instante da ocorrência da contingência. Ou seja, após a análise de investimentos realizada, torna-se necessário verificar através de simulação dinâmica, se a proposta de ajuste e investimento realizada é viável quanto ao limite de torque permitido nas máquinas do sistema. Em outras palavras, a potencia reativa alocada pode não ser adequada para o problema de impacto nos eixos dos geradores.

Uma proposta de incluir restrições de impactos nos eixos dos geradores em análises de sistemas elétricos pode ser encontrada em Moreira et. al. (2005) e Oliveira et. al. (2006). Estas referências apresentam, basicamente, uma metodologia para a redução do impacto nos eixos dos geradores durante o fechamento de anel elétrico no processo de recomposição

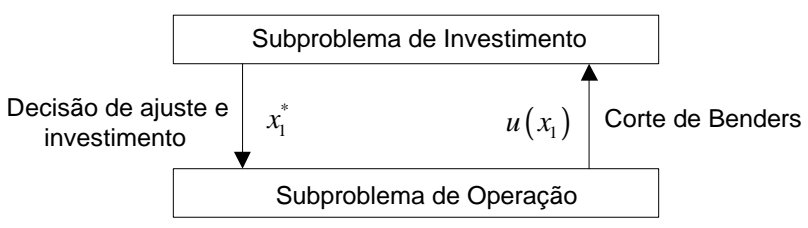

Figura 1: Decomposição hierárquica para planejamento.

do sistema. Os autores também utilizaram a técnica de Benders decompondo o problema em dois estágios: (i) análise estática do sistema antes do fechamento do anel; (ii) análise dinâmica do sistema no instante de fechamento do anel. O operador do sistema brasileiro (ONS) adota, para a maioria das usinas hidrelétricas, um limite de impacto nos eixos dos geradores em torno de $50 \%$ da capacidade nominal da máquina. Este limite é de fato uma medida de torção no eixo do gerador que depende da forma construtiva da máquina. Os dados de torque são difíceis de medir e não prontamente acessíveis. Adotar um único valor (50\%) para o limite dos torques de todos os geradores constitui-se em uma simplificação que os autores acreditam ser aceitável para o algoritmo proposto.

Neste sentido, o presente artigo propõe uma metodologia de inclusão de restrições de impactos nos eixos dos geradores ao tradicional problema de planejamento da operação do SEP. O objetivo desta proposta é minimizar os investimentos necessários para a operação viável do sistema em regime de contingência, mantendo-se os impactos nos geradores dentro dos limites recomendados pelo ONS. Estudos de casos são realizados para testar a metodologia proposta.

\section{METODOLOGIA PROPOSTA}

A metodologia proposta neste trabalho consiste em tratar o planejamento da operação do SEP através de uma nova técnica de decomposição que inclui um novo subproblema (subproblema-3) ao tradicional planejamento da operação, ou seja:

- Subproblema-1: Ou subproblema de investimento, considera o sistema em condições normais com o objetivo de minimizar os investimentos em suporte de potência reativa.

- Subproblema-2: Ou subproblema de operação, considera o sistema em regime de contingência com o objetivo de verificar se os investimentos e ajustes realizados no subproblema-1 são suficientes para uma operação viável.

- Subproblema-3: Ou subproblema de impacto, considera o sistema no instante em que ocorre a contingência com 


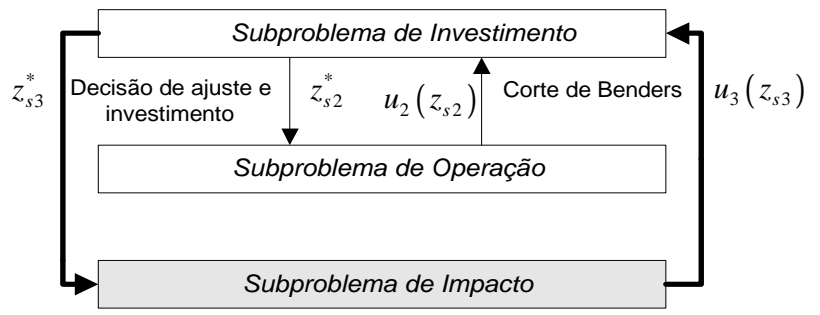

Figura 2: Metodologia de decomposição proposta.

o objetivo de verificar se os impactos nos eixos dos geradores encontram-se nos limites recomendados pelo ONS.

A Figura 2 ilustra este esquema de solução.

Nesta figura, $z_{s 2}=\left\{V_{g}, Q A_{i}, P G a_{i}, \alpha_{i}\right\}$ representa o conjunto das variáveis de ajuste, ou de decisão, do subproblema1 , consideradas como constantes no subproblema-2, ou seja, $z_{s 2}^{*}=\left\{V_{g}^{*}, Q A_{i}^{*}, P G a_{i}^{*}, \alpha_{i}^{*}\right\}$, onde:

$V_{g}$ Representa a tensão das barras de geração;

$Q A_{i}$ Representa a alocação de potência reativa na barra $i$, através da instalação de bancos de capacitores e/ou indutores;

$P G a_{i}$ Representa a geração de potência ativa na barra $i$;

$\alpha_{i}$ Representa o coeficiente associado ao corte de carga na barra $i$;

$u_{2}\left(z_{s 2}\right)$ Representa a restrição de corte de Benders gerada no subproblema-2.

Neste esquema, a solução do subproblema-1 fornece o mínimo investimento em suporte de potência reativa $\left(Q A_{i}^{*}\right)$ para que o subproblema-2 seja viável. Caso este subproblema não seja viável, é construída a restrição de corte de Benders $u_{2}\left(z_{s 2}\right)$.

$\mathrm{O}$ conjunto $z_{s 3}=\left\{E_{i}^{\prime \prime}, \delta_{i}, P G a_{i}, \alpha_{i}, V_{i}\right\}$, por sua vez, inclui as variáveis de decisão do subproblema-1, consideradas como constantes no subproblema-3, ou seja, $z_{s 3}^{*}=$ $\left\{E_{i}^{\prime \prime}{ }^{*}, \delta_{i}^{*}, P G a_{i}^{*}, \alpha_{i}^{*}, V_{i}^{*}\right\}$, onde:

$E_{i}^{\prime \prime}$ Representa a tensão interna da máquina localizada na barra $i$;

$\delta_{i}$ Representa o ângulo interno da máquina localizada na barra $i$;
$V_{i}$ Representa a tensão da barra $i$, cuja carga é modelada como impedância constante;

$u_{3}\left(z_{s 3}\right)$ Representa a restrição de corte de Benders gerada no subproblema-3.

A solução do subproblema-1 também fornece o mínimo corte de carga $\left(\alpha_{i}^{*}\right)$, necessário para que o impacto nos geradores não exceda o limite recomendado pelo ONS no subproblema3. Caso ocorra violação deste limite, é construída a restrição de corte de Benders $u_{3}\left(z_{s 3}\right)$.

Com as restrições de Benders incluídas a cada iteração, o subproblema-1 é executado novamente para o reajuste das variáveis de investimento $\left(z_{s 2}\right)$ e para reajuste do ponto de operação $\left(z_{s 3}\right)$.

O procedimento ilustrado na Figura 2 continua até que não ocorram violações do limite de impacto recomendado pelo ONS e que a operação do sistema seja viável, para todas as contingências da lista.

O Apêndice-A apresenta as formulações dos três subproblemas.

\section{ESTUDO DE CASOS}

Para mostrar as aplicações da ferramenta proposta, serão utilizados os sistemas IEEE 14 barras e IEEE 118 barras. As simulações foram conduzidas considerando-se todas as barras destes sistemas como candidatas à alocação de potência reativa. Os limites operacionais de tensão em todas as barras são 0,95 e $1,05 \mathrm{pu}$.

Estes sistemas foram considerados operando com carga pesada, a fim de determinar o investimento necessário em suporte de potência reativa sob condições extremas de carregamento. Foi considerado um percentual de $145 \%$ do carregamento nominal destes sistemas.

Os dados destes sistemas podem ser encontrados no endereço: www.ee.washington.edu/research/pstca.

A metodologia proposta neste artigo é testada através dos resultados obtidos nas duas condições de simulação a seguir:

Condição-1: A simulação é realizada de maneira tradicional, ou seja, sem considerar as restrições de impacto nos eixos dos geradores;

Condição-2: A simulação é realizada conforme metodologia proposta, ou seja, incluindo-se as restrições de impacto.

\section{1) Sistema IEEE-14:}

Este sistema possui 14 barras, 20 circuitos e 5 geradores. Os 
Tabela 1: Alocação de Potência Reativa: IEEE-14.

\begin{tabular}{|c|c|c|}
\hline & Condição-1 & Condição-2 \\
\hline Barra & $Q A(\mathrm{MVAr})$ & $Q A(\mathrm{MVAr})$ \\
\hline 13 & 2,33 & 4,16 \\
\hline
\end{tabular}

Tabela 2: Variação da potência na contingência 1:IEEE-14.

\begin{tabular}{|c|c|c|c|c|}
\hline & \multicolumn{2}{|c|}{ Condição-1 } & \multicolumn{2}{c|}{ Condição-2 } \\
\hline Barra & $\begin{array}{c}P G a^{*} \\
(\mathrm{MW})\end{array}$ & $\begin{array}{c}P G d \\
(\mathrm{MW})\end{array}$ & $\begin{array}{c}P G a^{*} \\
(\mathrm{MW})\end{array}$ & $\begin{array}{c}P G d \\
(\mathrm{MW})\end{array}$ \\
\hline 1 & 87,41 & 75,60 & 59,17 & 47,24 \\
\hline 2 & 221,64 & 191,08 & 236,40 & 208,34 \\
\hline $\mathbf{3}$ & $\mathbf{3 6 , 7 8}$ & $\mathbf{6 5 , 6 4}$ & $\mathbf{3 9 , 3 2}$ & $\mathbf{5 9 , 1 9}$ \\
\hline 6 & 2,29 & 4,50 & 12,52 & 14,22 \\
\hline 8 & 38,99 & 41,00 & 39,16 & 40,64 \\
\hline
\end{tabular}

Tabela 3: Ajuste das tensões dos geradores: IEEE-14.

\begin{tabular}{|c|c|c|}
\hline & Condição-1 & Condição-2 \\
\hline Barra & Tensão $(\mathrm{pu})$ & Tensão $(\mathrm{pu})$ \\
\hline 1 & 1,048 & 1,049 \\
\hline 2 & 1,049 & 1,045 \\
\hline $\mathbf{3}$ & $\mathbf{1 , 0 0 8}$ & $\mathbf{0 . 9 6 9}$ \\
\hline 6 & 1,050 & 1,050 \\
\hline 8 & 1,049 & 1,049 \\
\hline
\end{tabular}

Tabela 4: Fluxo de potência ativa contingência 1: IEEE-14.

\begin{tabular}{|c|c|c|}
\hline & Condição-1 & Condição-2 \\
\hline Circuito & Fluxo (MW) & Fluxo (MW) \\
\hline $2-3$ & 85,28 & 81,28 \\
\hline
\end{tabular}

dados associados aos geradores e a lista das contingências simuladas neste sistema encontram-se no Apêndice-B.

Considerando-se a condição-1 de simulação, foi necessária alocação de potência reativa $(Q A)$ no sistema para a operação viável do mesmo em regime de contingência, conforme a lista apresentada no Apêndice-B. Neste caso, a Tabela 1 mostra o valor da alocação necessária.

Este investimento em suporte de potência reativa torna viável a operação do sistema em regime de contingência. En- tretanto, no instante de ocorrência da contingência 1 , ocorre violação do limite de impacto $(P A)$ de geração na barra 3 . A Tabela 2 apresenta a variação instantânea da potência elétrica gerada nas barras de geração do sistema no instante de ocorrência da contingência 1. Verifica-se que na condição1, o impacto no gerador da barra 3 é de 28,86 MW, calculado pela diferença entre o valor obtido antes da contingência $\left(P G a_{3}^{*}=36,78 M W\right)$ e o valor obtido no instante da contingência $1\left(P G d_{3}=65,64 M W\right)$. Como a capacidade do gerador 3 é de $40 \mathrm{MW}$, ocorreu um excesso de impacto de $8,86 \mathrm{MW}$.

Já no instante de ocorrência da contingência 2, não houve violação de limite de impacto de geração no sistema.

Considerando-se a condição-2 de simulação, incluindo-se as restrições de impacto na análise conforme metodologia proposta, o impacto de geração na barra 3 passa a ser mantido nos limites recomendados pelo ONS para a contingência 1 .

Verificou-se que a inclusão das restrições dinâmicas no planejamento do sistema alterou significativamente o valor da alocação de potência reativa necessária para a operação viável do mesmo em regime permanente. Isto pode ser observado na condição-2 da Tabela 1 . Porém, observou-se que a localização da barra para investimento não foi alterada. Devido ao acréscimo de restrições ao problema na condição-2, houve um aumento do suporte de potência reativa necessário nesta condição.

A Tabela 2 permite verificar que o impacto de geração na barra 3 obtido na condição-2 é igual a 19,87 MW, ou seja, está dentro do limite recomendado de $20 \mathrm{MW}$.

Da Tabela 2 observa-se ainda que o despacho de geração dos outros geradores do sistema também foi alterado pela aplicação da metodologia proposta, sendo que a geração total permanece praticamente a mesma, tendo em vista que não ocorreu corte de carga.

A opção pelo corte de carga ou pelo redespacho de geração é realizada de maneira ótima pela metodologia proposta. Isto porque um custo é associado ao corte de carga, enquanto que o redepacho de geração não é penalizado. Desta forma, a metodologia prioriza o redespacho, optando pelo corte de carga somente quando este redespacho não for suficiente para atender ao critério de impacto definido pelo operador do sistema.

Além do investimento necessário em suporte de potência reativa, a inclusão das restrições dinâmicas também alterou o ajuste dos controles do sistema. A Tabela 3 apresenta o ajuste das tensões das barras de geração nas duas condições de simulação. Vale notar que a tensão da barra 3, cujo impacto de geração excedeu os limites, sofreu a maior alteração. 
Além disto, com a inclusão das restrições dinâmicas, o fluxo de potência ativa no circuito sob análise na contingência 1 (circuito 2-3) foi reduzido, como mostra a Tabela 4. Esta redução contribuiu para a eliminação da violação de impacto no gerador da barra 3 .

\section{2) Sistema IEEE-118:}

Este sistema possui 118 barras, 186 circuitos e 54 geradores. A lista de contingências simuladas neste sistema e os dados dos geradores que sofrem maior impacto devido à ocorrência destas contingências encontram-se no Apêndice-B.

As simulações realizadas utilizando-se este sistema foram conduzidas seguindo o mesmo enfoque abordado para o sistema IEEE-14.

A Tabela 5 apresenta os resultados de alocação de potência reativa nas duas condições de simulação propostas. Observase que a inclusão das restrições dinâmicas no planejamento do sistema, conforme condição-2 elevou o valor da alocação de potência reativa necessária para a operação viável do mesmo em regime de contingência. Porém, não alterou a localização da barra de investimento em suporte de potência reativa.

A Tabela 6 apresenta as maiores variações instantâneas da potência elétrica gerada nas duas condições de simulação propostas. As contingências 1 e 2 causaram impactos excessivos nos eixos dos geradores. As maiores violações ocorreram na barra 4 e na barra 61 . Neste caso, o impacto na barra 4 foi devido a contingência 1, enquanto que a contingência 2 causou maior impacto na barra 61. Já na condição-2, as restrições de impacto nos geradores foram atendidas. Para eliminar as violações de impacto ocorridas nas contingências 1 e 2, houve um redespacho de geração, como pode ser observado na Tabela 6. O redespacho em cada barra é necessário para eliminar a violação de impacto causada pela respectiva contingência.

Além da modificação em investimento em suporte de potência reativa, a inclusão das restrições dinâmicas também alterou o ajuste dos controles do sistema.

A Tabela 7 apresenta o ajuste das tensões das barras de geração nas duas condições de simulação.

Além disto, com a inclusão das restrições dinâmicas, o fluxo de potência ativa nos circuitos em contingência nos casos 1 e 2, circuitos 8-5 e 64-65 respectivamente, foram reduzidos, como mostra a Tabela 8. Estas reduções contribuíram para a eliminação das violações de impacto ocorridas em função destas contingências. Verificou-se, portanto, que a aplicação da metodologia proposta limitou os fluxos nos circuitos nos níveis adequados para evitar violações de impacto nos gera-
Tabela 5: Alocação de Potência Reativa: IEEE-118.

\begin{tabular}{|c|c|c|}
\hline & Condição-1 & Condição-2 \\
\hline Barra & $Q A(\mathrm{MVAr})$ & $Q A(\mathrm{MVAr})$ \\
\hline 53 & 8,17 & 12,12 \\
\hline
\end{tabular}

Tabela 6: Variação da potência no caso 1: IEEE-118.

\begin{tabular}{|c|c|c|c|c|}
\hline & \multicolumn{2}{|c|}{ Condição-1 } & \multicolumn{2}{c|}{ Condição-2 } \\
\hline \multirow{2}{*}{ Barra } & $\begin{array}{c}P G a^{*} \\
(\mathrm{MW})\end{array}$ & $\begin{array}{c}P G d \\
(\mathrm{MW})\end{array}$ & $\begin{array}{c}P G a^{*} \\
(\mathrm{MW})\end{array}$ & $\begin{array}{c}P G d \\
(\mathrm{MW})\end{array}$ \\
\hline 4 & 62,42 & 121,96 & 67,04 & 101,74 \\
\hline 6 & 98,12 & 160,16 & 102,84 & 149,52 \\
\hline 8 & 187,95 & 69,37 & 150,91 & 48,25 \\
\hline 12 & 63,07 & 106,31 & 67,08 & 100,53 \\
\hline 59 & 68,92 & 125,81 & 70,97 & 106,80 \\
\hline 61 & 103,18 & 185,19 & 106,80 & 159,36 \\
\hline
\end{tabular}

Tabela 7: Ajuste das tensões dos geradores: IEEE-118.

\begin{tabular}{|c|c|c|}
\hline & Condição-1 & Condição-2 \\
\hline Barra & Tensão $(\mathrm{pu})$ & Tensão $(\mathrm{pu})$ \\
\hline 4 & 1,015 & 1,002 \\
\hline 6 & 1,008 & 1,002 \\
\hline 8 & 1,016 & 1,027 \\
\hline 12 & 0,999 & 0,995 \\
\hline 59 & 1,044 & 0,990 \\
\hline 61 & 1,047 & 1,002 \\
\hline
\end{tabular}

Tabela 8: Fluxo de potência na contingência 1: IEEE-118.

\begin{tabular}{|c|c|c|}
\hline & Condição-1 & Condição-2 \\
\hline Circuito & Fluxo (MW) & Fluxo (MW) \\
\hline $8-5$ & 336,71 & 289,63 \\
\hline $64-65$ & 366,80 & 335,48 \\
\hline
\end{tabular}

dores, sem a necessidade de utilização de restrições de fluxo mos circuitos.

As Figuras 3 e 4 mostram os resultados de simulações dinâmicas realizadas com o programa ANATEM (Rangel et. al., 2000), antes do redespacho determinado na Tabela 6. A continuidade das curvas é apresentada de modo a facilitar o en- 


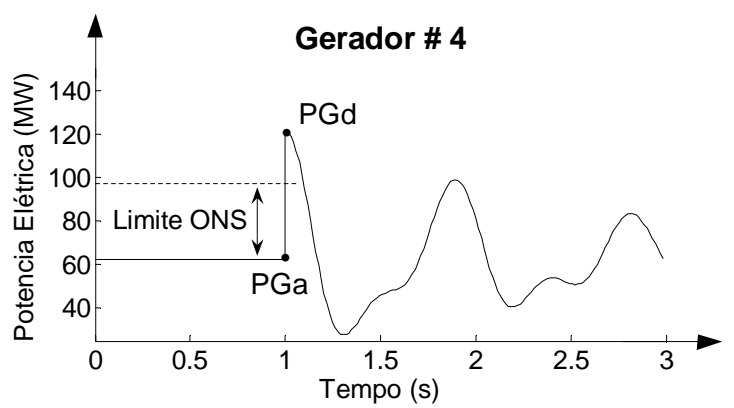

Figura 3: Gerador \# 4, Contingência 1, Condição 1.

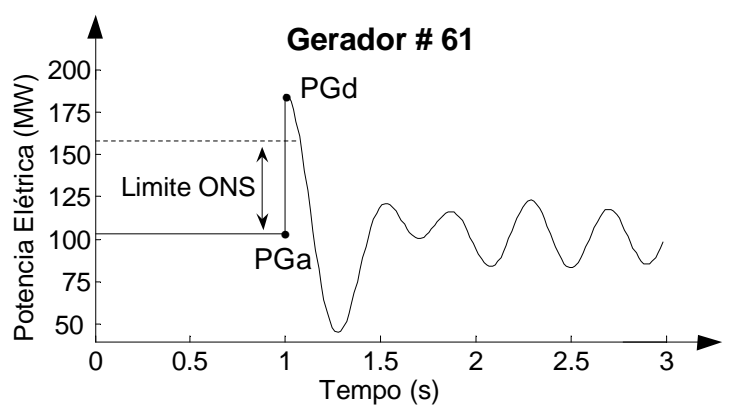

Figura 4: Gerador \# 61, Contingência 2, Condição 1.

tendimento. Para tanto, adotou-se para a constante de inércia das máquinas, $\mathrm{H}=5 \mathrm{~s}$ e constante de amortecimento, $\mathrm{D}=5$. Os reguladores não foram considerados nas simulações porque eles não influenciam no instante de análise de impacto.

As Figuras 5 e 6 mostram as simulações dinâmicas realizadas após o redespacho apresentado na Tabela 6. Pode-se observar que os impactos permanecem dentro dos limites, comprovando a precisão da modelagem proposta.

\section{CONCLUSÃo}

Este artigo apresentou uma proposta de inclusão de restrições dinâmicas no problema de planejamento da operação de sistemas elétricos de potência. A inclusão destas restrições possibilitou manter o impacto nos eixos dos geradores nos limites recomendados pelo ONS, durante a ocorrência de contingências no SEP. Para tanto, o valor do suporte de potência reativa foi alterado. Adicionalmente, a aplicação da metodologia proposta reduziu os fluxos de potência em alguns circuitos, resultando na redução do impacto nos geradores no instante de retirada destes circuitos. A metodologia proposta permitiu a operação viável do sistema em regime permanente, atendendo restrições dinâmicas no instante de contingência, mostrando-se como uma valiosa ferramenta para o planejamento da operação dos SEP.

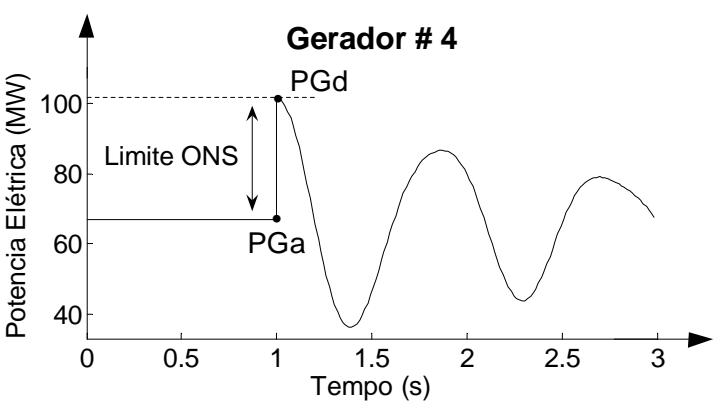

Figura 5: Gerador \# 4, Contingência 1, Condição 2.

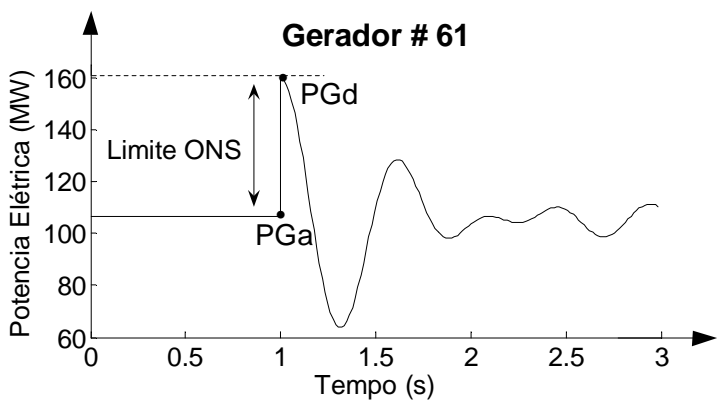

Figura 6: Gerador \# 61, Contingência 2, Condição 2.

\section{AGRADECIMENTOS}

O primeiro e o último autor gostariam de agradecer ao apoio financeiro do CNPq.

\section{REFERÊNCIAS}

Monticelli, A.; Pereira M. V. F. and Granville S. (1987). Security-constrained optimal power flow with postcontingency corrective rescheduling. IEEE Transactions on Power Systems, Vol. II, No.1.

Granville, S.; Pereira, M. V. P. and Monticelli, A. (1988). An integrated methodology for VAr sources planning. IEEE Transactions on Power Systems, Vol. III, No. 2, pp. 549-557.

Vaahedi, E.; Tamby, J.; Mansour, Y.; Wenyuan, L. and Sun, D. (1999). Large scale voltage stability constrained optimal VAr planning and voltage stability applications using existing OPF/optimal VAr planning tools. IEEE Transactions on Power Systems, Vol. XIV, No. 1, pp. 65-74.

Benders, J. F. (1962). Partitioning procedure for solving mixed variables programming problems, Numerishe Mathematics, pp. 238-262. 
Granville, S. and Abib Lima, M. C. (1994). Application of decomposition techniques to VAr planning: methodological and computational aspects. IEEE Transactions on Power Systems, Vol. IX, No. 4, pp. 1780-1787.

Vaahedi, E.; Mansour, Y.; Fuchs, C.; Granville, S.; Latore, M. D. L. and Hamadanizadeh, H. (2001). Dynamic security constrained optimal power flow/VAr planning. IEEE Transactions on Power Systems, Vol. XVI, No. 1, pp. 38-43.

de Oliveira, L. W.; de Oliveira, E. J.; Pereira, J. L. R.; Cordeiro, L. A. and Passos Filho, J. A. (2005). Proposta de ajuste da tensão de referência do compensador estático de reativos, Sixth Latin-American Congress: Electricity, Generation and Transmission, No. A-124.

Geoffrion, H. M. (1972). Generalized Benders Decomposition, JOTA, Vol. X.

Moreira, W. C.; de Oliveira, E. J.; Martins, N.; Pereira, J. L. R. and de Oliveira, L. W. (2005). Impacto em máquinas síncronas durante o fechamento de anel na recomposição de sistemas de potência, VI SIMPASE (Simpósio de Automação de Sistemas Elétricos), No. 36.

de Oliveira, E. J.; Martins, N.; Fontoura, R. M. and Pereira, J. L. R. (2006). Fluxo de potência ótimo com restrições de impactos durante o fechamento de anel, Proceedings of the X SEPOPE (Symposium of Specialists in Electric Operational and Expansion Planning), No. SP-035.

ONS - Brazilian System Operator. Guidelines and Criteria for Electrical Studies - Guidelines and Criteria for loop closure, item 8.5, sub-module 23.3. (In Portuguese). http://www.ons.org.br/ons/procedimentos/index.htm.

Rangel, R. D.; Júnior, S. G.; Guimarães, C.H.C.; Martins, N.; Castro, A.; Pinto, H. J. C. P. and Carvalho, A. R. C. D. (2000). Recent Developments in ANATEM - A Comprehensive Program for the Analysis of Electromechanical Stability of Large Power Systems, Proceedings of the VII SEPOPE (Symposium of Specialists in Electric Operational and Expansion Planning).

Wright, S. J. (1997). Primal-Dual Interior Point Methods, Society for Industrial and Applied Mathematics (SIAM).

Castronuovo, E. D. (2001). Aplicação do método de pontos interiores no fluxo de potência ótimo não linear com utilização de processamento de alto desempenho, Tese de doutorado, UFSC.

Yuan, Y.; Kubokawa, J. and Sasaki, H. (2003). A solution of optimal power flow with multicontingency transient stability constraints. IEEE Transactions on Power Systems, Vol. XVIII, No. 3, pp. 1094-1102.
Deqiang, G.; Thomas, R. J. and Zimmerman, R. D. (2000). Stability-constrained optimal power flow. IEEE Transactions on Power Systems, Vol. XV, No. 2, pp. 535540.

Granville, S.; Mello, J. C. O. and Melo, A. C. G. (1996). Aplication of interior point methods to power flow unsolvability. IEEE Transactions on Power Systems, Vol. XI, No. 2, p.p. 1096-1103.

Torres, G. L.; Quintana, V. H. and de Carvalho, M. A. (2002). Higher-order interior-point methods for computing minimum load shedding and maximum loadability of power systems, Proceedings of the VIII SEPOPE (Symposium of Specialists in Electric Operational and Expansion Planning), No. IP-057.

\section{APÊNDICE A}

A seguir, serão apresentadas as formulações dos três subproblemas considerados. Cada um destes subproblemas consiste em um problema de programação não-linear. Para a sua solução, utilizou-se o Método Primal-Dual de Pontos Interiores (Wright, 1997; Castronuovo, 2001).

\section{1) Subproblema-1: Investimento:}

Este subproblema é modelado como:

$$
\operatorname{Min}\left(\sum_{i \in C} c_{Q A} \cdot Q A_{i}+\sum_{i \in S} c_{L S} \cdot \alpha_{i}\right)
$$

$\mathrm{sa} /$

$$
\begin{gathered}
P G a_{i}-\left(1-\alpha_{i}\right) \cdot P L_{i}-\sum_{j \in \Omega i} P_{i j}=0 \\
Q G_{i}+Q A_{i}-\left(1-\alpha_{i}\right) \cdot Q L_{i}-\sum_{j \in \Omega i} Q_{i j}=0 \\
P G a_{i} \cdot X d_{i}^{\prime \prime}-E_{i}^{\prime \prime} \cdot V_{i} \cdot \operatorname{sen}\left(\delta_{i}-\theta_{i}\right)=0 \\
Q G_{i} \cdot X d_{i}^{\prime \prime}-E_{i}^{\prime \prime} \cdot V_{i} \cdot \cos \left(\delta_{i}-\theta_{i}\right)+V_{i}^{2}=0 \\
P G_{i}^{\min } \leq P G a_{i} \leq P G_{i}^{\max } \\
Q G_{i}^{\min } \leq Q G_{i} \leq Q G_{i}^{\max } \\
Q A_{i}^{\min } \leq Q A_{i} \leq Q A_{i}^{\max } \\
V_{i}^{\min } \leq V_{i} \leq V_{i}^{\max } \\
0 \leq \alpha_{i} \leq 1
\end{gathered}
$$




$$
\begin{aligned}
& \sum_{m \in L}\left[\sum_{s \in \Omega z_{S 2}}\left[\sum_{r \in \Omega z_{R 2}}\left(\pi_{m r 2} \cdot E z_{s 2 r}\right) z_{s 2}\right]\right] \\
& +\sum_{m \in L}\left[\sum_{s \in \Omega z_{S 3}}\left[\sum_{r \in \Omega z_{R 3}}\left(\pi_{m r 3} \cdot E z_{s 3 r}\right) z_{s 3}\right]\right] \geq \\
& \sum_{m \in L}\left[w_{m 2}+\sum_{s \in \Omega z_{S 2}}\left[\sum_{r \in \Omega z_{R 2}}\left(\pi_{m r 2} \cdot E z_{s 2 r}\right) z_{s 2}^{*}\right]\right] \\
& +\sum_{m \in L}\left[w_{m 3}+\sum_{s \in \Omega z_{S 3}}\left[\sum_{r \in \Omega z_{R 3}}\left(\pi_{m r 3} \cdot E z_{s 3 r}\right) z_{s 3}^{*}\right]\right]
\end{aligned}
$$

Onde:

$P L_{i}$ Representa a carga ativa na barra $i$;

$Q L_{i}$ Representa a carga reativa na barra $i$

$c_{L S}$ Representa o custo de corte de carga mantendo-se o fator de potência constante;

$c_{Q A}$ Representa o custo de alocação de potência reativa;

$S$ Representa o conjunto de barras candidatas a receber corte de carga;

$C$ Representa o conjunto de barras candidatas à alocação de potência reativa.

As equações (2) e (3) são relativas aos fluxos de carga ativa e reativa na barra $i$, respectivamente, onde:

$P_{i j}$ Representa o fluxo de potência ativa no ramo $i-j$;

$\Omega_{i}$ Representa o conjunto de barras adjacentes à barra $i$;

$Q G_{i}$ Representa a potência reativa gerada na barra $i$;

$Q_{i j}$ Representa o fluxo de potência reativa no ramo $i-j$.

As equações (4) e (5) são associadas ao modelo do gerador, ou seja, uma fonte de tensão $\left(E^{\prime \prime} \angle \delta\right)$ atrás da reatância subtransitória ( $\left.X d^{\prime \prime}\right)$ (Yuan et. al., 2003; Deqiang et. al., 2000), onde:

$\theta_{i}$ Ângulo nodal da barra $i$.

As equações (6) a (10) correspondem às restrições de canalização do problema, onde:
$P G_{i}^{\text {min }}, P G_{i}^{\max }$ Representam os limites de geração de potência ativa na barra $i$;

$Q G_{i}^{\min }, Q G_{i}^{\max }$ Representam os limites de geração de potência reativa na barra $i$;

$Q A_{i}^{\min }, Q A_{i}^{\max }$ Representam os limites de alocação de potência reativa na barra $i$;

$V_{i}^{\min }, V_{i}^{\max }$ Representam os limites de tensão da barra $i$.

A Equação (11) corresponde à restrição de Benders, onde:

$L$ Representa a lista de contingências;

$\Omega z_{S 2}$ Representa o conjunto de variáveis de decisão $z_{s 2}=$ $\left\{V_{g}, Q A_{i}\right\}$ do subproblema-2;

$\Omega z_{S 3}$ Representa o conjunto de variáveis de decisão $z_{S 3}=$ $\left\{E_{i}^{\prime \prime}, \delta_{i}, P G_{i}, \alpha_{i}, V_{i}\right\}$ do subproblema-3;

$\Omega z_{R 2}$ Representa o conjunto de restrições do subproblema-2 associadas $\operatorname{com} z_{s 2}^{*}$;

$\Omega z_{R 3}$ Representa o conjunto de restrições do subproblema-3 associadas $\operatorname{com} z_{s 3}^{*}$;

$w_{m 2}$ Valor da função objetivo do subproblema-2, para a contingência $m$;

$w_{m 3}$ Valor da função objetivo do subproblema-3, para a contingência $m$;

$E z_{s 2 r}$ Derivada parcial da restrição rem relação a variável $z_{s 2}$

$E z_{s 3 r}$ Derivada parcial da restrição $r$ em relação a variável $z_{s 3}$

$\pi_{m r 2}$ Multiplicador de Lagrange obtido do subproblema-2 associado com a restrição $r$, para a contingência m;

$\pi_{m r 3}$ Multiplicador de Lagrange obtido do subproblema-3 associado com a restrição $r$, para a contingência $m$.

A Equação (11) correspondente à restrição de Benders só é incluída ao subproblema-1 após as execuções do subproblema-2 e do subproblema-3.

A Equação (1) mostra que um novo termo foi incluído à função objetivo do tradicional problema de planejamento da operação. No original problema da operação de SEP, o objetivo é somente minimizar o investimento em suporte de potência reativa, ou seja, $\operatorname{Min} \sum_{i \in C} c_{Q A} \cdot Q A_{i}$. Desta forma, o novo termo incluído é associado à minimização de corte de carga no sistema, dado por $\operatorname{Min} \sum_{i \in S} c_{L S} \cdot \alpha_{i}$ (Granville et. 
al., 1996; Torres et. al., 2002). Este corte de carga pode ser necessário devido à inclusão de severas restrições dinâmicas no problema.

Neste subproblema, o custo de investimento em suporte de potência reativa é linear, pois ele representa o custo real de investimento em capacitor e reator. Para tanto, foram necessárias duas variáveis de alocação de potência reativa $\left(Q A_{i}\right)$, sendo uma para alocação capacitiva e a outra para alocação indutiva. $\mathrm{O}$ valor do custo $c_{Q A}$ foi o mesmo tanto para o investimento em banco de capacitores quanto para o investimento em reatores.

Sendo assim, o planejamento da operação do SEP torna-se um problema de otimização multi-objetivo, devido à inclusão de restrições dinâmicas na análise.

\section{2) Subproblema-2: Operação:}

Este subproblema considera o SEP em regime de contingência, sendo modelado para cada contingência como:

$$
\operatorname{Min} \sum_{i \in B} \frac{1}{2} \cdot c_{Q A f} \cdot Q A f_{i}^{2}
$$

$\mathrm{sa} /$

$$
\begin{gathered}
P G_{i}-\left(1-\alpha_{i}^{*}\right) \cdot P L_{i}-\sum_{j \in \Omega i} P_{i j}=0 \\
Q G_{i}+Q A f_{i}-\left(1-\alpha_{i}^{*}\right) \cdot Q L_{i}+Q A_{i}^{*}-\sum_{j \in \Omega i} Q_{i j}=0 \\
P G_{i}^{\min } \leq P G_{i} \leq P G_{i}^{\max } \\
Q G_{i}^{\min } \leq Q G_{i} \leq Q G_{i}^{\max } \\
V_{i}^{\min } \leq V_{i} \leq V_{i}^{\max }
\end{gathered}
$$

Onde:

$Q A f_{i}$ Representa a alocação fictícia de potência reativa na barra $i$

$c_{Q A f}$ Representa o custo de alocação fictícia de potência reativa na barra $i$;

$B$ Representa o conjunto de todas as barras do sistema;

$P G_{i}$ Representa a geração de potência ativa na barra $i$, calculada no subproblema de operação.

Neste subproblema, todas as barras são especificadas como candidatas à alocação fictícia de potência reativa $(Q A f)$. Isto é necessário para garantir a convergência do problema caso a contingência seja inviável. A função objetivo de custo quadrática, Equação (12), foi utilizada pois ela representa um custo fictício de investimento, não havendo necessidade de distinção entre capacitor e reator.

Quando $\sum_{i \in B} Q A f_{i}=0$, significa que a contingência em análise é viável. No entanto, se $\sum_{i \in B} Q A f_{i} \neq 0$ a operação do sistema em regime desta contingência ainda é inviável. Neste caso, deve-se construir a restrição de corte de Benders conforme Equação (11).

\section{3) Subproblema-3: Impacto:}

Este subproblema considera o SEP no instante do impacto sendo modelado para cada contingência como:

$$
\operatorname{Min} \sum_{i \in G} \frac{1}{2} \cdot c_{P A} \cdot P A_{i}^{2}
$$

$\mathrm{sa} /$

$$
P G d_{i}+P A_{i}-\left(1-\alpha_{i}^{*}\right) \cdot P L_{i}-\sum_{j \in \Omega i} P_{i j}=0
$$

$$
\begin{gathered}
Q G_{i}-\left(1-\alpha_{i}^{*}\right) \cdot Q L_{i}-\sum_{j \in \Omega i} Q_{i j}=0 \\
\left(P G d_{i}+P A_{i}\right) \cdot X d_{i}^{\prime \prime}-E_{i}^{\prime \prime} * V_{i} \cdot \operatorname{sen}\left(\delta_{i}^{*}-\theta_{i}\right)=0 \\
Q G_{i} \cdot X d_{i}^{\prime \prime}-E_{i}^{\prime \prime}{ }^{*} \cdot V_{i} \cdot \cos \left(\delta_{i}^{*}-\theta_{i}\right)+V_{i}^{2}=0 \\
\left|P G d_{i}-P G a_{i}^{*}\right| \leq 50 \% \cdot P G n_{i} \\
P L_{i}=\left(1-\alpha_{i}^{*}\right) \cdot P L_{i}^{0} \cdot\left(V_{i}\right)^{2} /\left(V_{i}^{*}\right)^{2} \\
Q L_{i}=\left(1-\alpha_{i}^{*}\right) \cdot Q L_{i}^{0} \cdot\left(V_{i}\right)^{2} /\left(V_{i}^{*}\right)^{2}
\end{gathered}
$$

Onde:

$P A_{i}$ Representa a injeção fictícia de potência ativa na barra $i$;

$c_{P A}$ Representa o custo de injeção fictícia de potência ativa na barra $i$;

$P G d_{i}$ Representa a geração de potência ativa na barra $i$, calculada no subproblema de impacto;

$P G n_{i}$ Representa a potência ativa nominal do gerador da barra $i$;

$P L_{i}^{0}$ Representa o valor original da carga ativa na barra $i$;

$Q L_{i}^{0}$ Representa o valor original da carga reativa na barra $i$. 
A função objetivo deste subproblema é associada à injeção fictícia de potência ativa $(P A)$ nas barras com geradores. Esta injeção é necessária para garantir a convergência do mesmo, caso o impacto nestes geradores ultrapasse o limite recomendado pelo ONS (Moreira et. al., 2005; Oliveira et. al., 2006).

A Equação (23) corresponde ao limite do ONS para a máquina $i$ no instante da contingência. Ou seja, a variação da potência elétrica gerada no instante do impacto deve ser inferior a $50 \%$ da capacidade nominal da máquina $\left(P G n_{i}\right)$.

As equações (24) e (25) correspondem ao modelo de carga como impedância constante utilizado neste subproblema.

Neste subproblema não são considerados os limites de tensão das barras do sistema $\left(V_{i}^{\text {min }} \leq V_{i} \leq V_{i}^{\text {max }}\right)$, pois no instante do impacto, os módulos das tensões nodais $\left(V_{i}\right)$ apresentam grandes variações. No entanto, como estas variações são de pequena duração, as mesmas são aceitáveis pelo operador do sistema e os reguladores de tensão não atuam no instante do impacto.

Quando $\sum_{i \in G} P A_{i}=0$, significa que o impacto em todos os geradores está dentro do limite recomendado pelo ONS. No entanto, se $\sum_{i \in G} P A_{i} \neq 0$ o impacto em um dos geradores ultrapassou o limite. Neste caso, deve-se construir a restrição de corte de Benders conforme Equação (11) e executar novamente o subproblema-1 juntamente com esta restrição.

A convergência global do problema proposto ocorre quando a operação do sistema em contingência é viável, e os impactos nos geradores devido às contingências estão dentro dos limites recomendados pelo ONS.

Ressalta-se que a execução do subproblema de investimento (subproblema-1) contém informações do tradicional problema de planejamento da operação do SEP, associadas às informações dinâmicas de impactos nos eixos dos geradores.

\section{APÊNDICE B}

A Tabela 9 apresenta os dados dos geradores do sistema IEEE-14. A Tabela 10 apresenta a lista de contingências do sistema IEEE-14.

A Tabela 11 apresenta a lista de contingências do sistema IEEE-118. A Tabela 12 apresenta a capacidade dos geradores que sofreram maior impacto devido a ocorrência das contingências desta lista.
Tabela 9: Dados dos Geradores: IEEE-14.

\begin{tabular}{|c|c|c|c|}
\hline Gerador & $\begin{array}{c}P G^{\max } \\
(\mathrm{MW})\end{array}$ & $\begin{array}{c}Q G^{\mathrm{min}} \\
(\mathrm{MVAr})\end{array}$ & $\begin{array}{c}Q G^{\max } \\
(\mathrm{MVAr})\end{array}$ \\
\hline 1 & 250,0 & $-50,0$ & 50,0 \\
\hline 2 & 250,0 & $-40,0$ & 50,0 \\
\hline 3 & 40,0 & 0 & 40,0 \\
\hline 4 & 40,0 & $-6,0$ & 24,0 \\
\hline 5 & 40,0 & $-6,0$ & 24,0 \\
\hline
\end{tabular}

Tabela 10: Lista de Contingências: IEEE-14.

\begin{tabular}{|c|c|c|}
\hline No & Retirada & Local \\
\hline 1 & Linha & $2-3$ \\
\hline 2 & Linha & $6-13$ \\
\hline
\end{tabular}

Tabela 11: Lista de Contingências: IEEE-118.

\begin{tabular}{|c|c|c|}
\hline No & Retirada & Local \\
\hline 1 & Linha & $8-5$ \\
\hline 2 & Linha & $64-65$ \\
\hline 3 & Linha & $53-54$ \\
\hline
\end{tabular}

Tabela 12: Dados de Geração: IEEE-118.

\begin{tabular}{|c|c|}
\hline Gerador & $\begin{array}{c}P G^{\max } \\
(\mathrm{MW})\end{array}$ \\
\hline 4 & 72,0 \\
\hline 6 & 108,0 \\
\hline 8 & 217,0 \\
\hline 12 & 72,0 \\
\hline 59 & 72,0 \\
\hline 61 & 108,0 \\
\hline
\end{tabular}

